\title{
Human Liver Specific Transcriptional Factor TCP10L Binds to MAD4
}

\author{
Dao-Jun Jiang, Hong-Xiu Yu, Sa-Yin Hexige, Ze-Kun Guo, Xiang Wang, Li-Jie Ma, \\ Zheng Chen, Shou-Yuan Zhao and Long Yu* \\ State Key Laboratory of Genetic Engineering, Institute of Genetics, School of Life Science, Fudan University, \\ Shanghai 200433, P. R. China
}

Received 6 August 2003, Accepted 9 September 2003

\begin{abstract}
A human gene T-complex protein 10 like (TCP10L) was cloned in our lab. A previous study showed that it expressed specifically in the liver and testis. A transcription experiment revealed that TCP10L was a transcription factor with transcription inhibition activity. In this study, the human MAD4 was identified to interact with TCP10L by a yeast two-hybrid screen. This finding was confirmed by immunoprecipitation and subcellular localization experiments. As MAD4 is a member of the MAD family, which antagonizes the functions of MYC and promotes cell differentiation, the biological function of the interaction between TCP10L and MAD4 may be to maintain the differentiation state in liver cells. Also, we propose that the up-regulation of $M y c$ is caused by the down-regulation of TCP10L in human hepatocarcinomas.
\end{abstract}

Keywords: MAD4, TCP10L, Yeast two-hybrid screen

\section{Introduction}

Liver cancer is a common disease that preferentially occurs in Africa and Asia. Based on 1990 data, liver cancer is ranked sixth in a list of the estimated number of cancer cases in the world (Pisani et al., 1997). Because of its poor prognosis and treatment, liver cancer claims an even higher proportion of all cancer deaths and was ranked in 1985 as the fourth most common cause of cancer mortality worldwide (Pisani et al., 1993). The progress of the prognosis and treatment on liver

\footnotetext{
Abbreviations: SDS-PAGE, sodium dodecyl sulphate polyacrylamide gel electrophoresis; EGFP, enhanced green fluorescent protein; RT-PCR, reverse transcriptase polymerase chain reaction; HA tag, haemagglutinin tag; MXI1, MAX interacting protein 1; bp, base pair; $\mathrm{h}$, hour; min, minute.
}

*To whom correspondence should be addressed.

Tel: 86-21-65642422; Fax: 86-21-65643250

E-mail: longyu@fudan.edu.cn cancer is largely dependent on the basic research on the gene regulation in the liver undergoing tumorigenesis. Transcription factors, especially those specifically expressed in the liver, may play a key role in hepatocarcinogenesis.

The Myc/Max/Mad transcription factor network is profoundly involved in the regulation of cell proliferation and differentiation (Baudino and Cleveland, 2001). Each member of the network contains a conserved basic Helix-Loop-Helix leucine zipper motif (bHLHZip) that facilitates protein-protein heterodimerization and protein-DNA binding. MYC and MAD proteins are functionally antagonized through competitively heterodimerizing with MAX and binding to DNA at the same consensus sequence CACGTG (E box). MYC:MAX complexes can activate transcription when bound to DNA at the E-box and promote cell proliferation and transformation (Henriksson and Luscher, 1996; Facchini and Penn, 1998; Dang, 1999). In contrast to MYC, the MAD family proteins (MAD1, MXIl, MAD3, and MAD4) inhibit cell proliferation and transformation MYC functions and promote cell differentiation (Ayer et al., 1993; Hurlin et al., 1995). In the past decade, strong associations between oncogenesis and transcription factors possessing the leucine zipper motif have been revealed (Smith et al., 1990; Amati et al., 1993; Luscher and Larsson, 1999).

A novel human gene T-complex 10 like (TCP1OL) that is down-regulated in liver hepatoma tissues was cloned in our lab. Its homologs in mice belong to the TCP10 family. One member of this family, $T C P 10 b^{t}$, was once considered a candidate of the T-complex responder for transmission ratio distortion (Schimenti et al., 1988; Cebra-Thomas et al., 1991). $T C P 1 O b^{t}$ is exclusively expressed in testicular germ cells (Ewulonu et al., 1993); however, the expression pattern of $T C P 10 L$ is different from that of $T C P 10 b^{t}$. Northern hybridization revealed that $T C P 10 L$ expressed specifically in the human liver and testis among 30 tissues that were examined. A subsequent analysis, based on the dual luciferase assay system, showed that the recombinant TCP10L was a transcription factor with transcriptional inhibition activity (Chen et al., 2003, in press). However, there is no reported 
function on any member of the TCP10 family in mice. The specific expression pattern and transcriptional inhibition activity indicate that TCP1OL might play an important role in the liver and testis, but how its function is exerted in vivo is unclear. From previous knowledge, transcription factors often interact with other regulatory members and form transcription complexes to perform their functions. Therefore, we utilized the yeast two-hybrid system to screen the proteins that may interact with TCP1OL. In this way, we hope to find the clue that can elucidate the mechanism of its potential function on gene transcription.

\section{Materials and Methods}

Materials The HEK293 cell line was purchased from ATCC (Manassas, USA) and the yeast strain EGY48(p8op-lacZ) was from CLONTECH (Franklin Lakes, USA). Matchmaker LexA twohybrid system, human liver cDNA library, and vectors of pLexA, pB42AD, pCMV-HA, pCMV-Myc, and pEGFP were products of CLONTECH. The pCDNA3.1/Myc-His(-)B vector was purchased from Invitrogen (Calsbad, USA). The recombinant plasmid pGEMT/TCP10L, which contained the coding region of TCP10L, was constructed in our lab (Chen et al., 2003 J. Human Genet., in press). Restriction endonucleases, such as EcoRI and XhoI, were products of New England Biolabs Inc. (Beverly, USA). The mouse anti-HA monoclonal antibody ( $\mathrm{HA} \mathrm{mAb}$ ) and mouse anti-Myc monoclonal antibody (Myc mAb) were purchased from CLONTECH. Cy5 (a cyanine-conjugated goat anti-mouse $\mathrm{IgG}$ ) was purchased from Molecular Research Center Inc. (Cincinnati, USA). The HRPconjugated anti-mouse antibody and protein G/protein A agarose beads were products of EMD Biosciences Inc. (Darmstadt, Germany).

Yeast two-hybrid screening A yeast two-hybrid assay was performed with the Matchmaker LexA two-hybrid system, according to the instructions from CLONTECH. To construct the bait plasmids, the full-length ( 1 to $645 \mathrm{bp}$ ), the front half (head, 1 to $315 \mathrm{bp}$ ), and the back half (tail, 307 to $645 \mathrm{bp}$ ) of the coding region of TCP10L cDNA were produced by PCR with pGEM-T/TCP10L as the template. The primers for the full-length coding region (P1: 5'-CGGAATTCATGCTGGCAGGTCAACTC-3', P2: 5'-TAAACT CGAGTCAGACACCCCCCC-3'), head region (P1, P3: 5'-GACC TCGAGTTACTTCCTGGCTTTCC-3') and tail region (P4: 5'-GC CGAATTCGCCAGGAAGAAATCTGC-3',P2) were designed to contain the EcoR I and Xho I restriction sites in the 5'-end and 3'end, respectively. The PCR products were digested with EcoR I$X h o I$ and then subcloned in frame into the same restriction sites in the DNA-binding domain of LexA in pLexA to give pLexA/ TCP10L, pLexA/TCP10L-H (Head), and pLexA/TCP10L-T (Tail) separately. The inserted cDNA in the library was cloned into the LexA transcription activation domain in the $\mathrm{pB} 42 \mathrm{AD}$ vector. The human liver cDNA library was screened. TCP10L was used as a bait to screen the reporter genes $L E U 2$ and lacZ. Transformed yeast was grown on plates that lacked both histidine and tryptophan. Individual cDNA of interest was purified from the positive clones and re-transformed into yeast for confirmation.
In vivo interaction assay The interaction between TCP10L and Mad4 in vivo was performed in HEK293 cells. Recombinant plasmid pCMV-HA/TCP10L was constructed with primers (P5: 5'ATCGAATTCACATGCTGGCAGGTC-3', P6: 5'-AATGGTACC TCAGACACCCCCC-3') and pGEM-T/TCP10L as the template. The open-reading frame of Mad4 was directly cloned from the human liver cDNA library with primers (P7: 5'-ACGGAATTCCA ATGGAGCTGAACTCC-3', P8: 5'-AATGGTACCCTACGAGAG GGCGGG-3'), then ligated into the pCMV-Myc vector to give pCMV-Myc/Mad4.

HEK293 cells were cultured in a DMEM medium that was supplemented with $10 \%$ bovine serum. The $2 \times 10^{5}$ HEK293 cells were seeded on the $35 \mathrm{~mm}$ disk and maintained at $37^{\circ} \mathrm{C}$ in a $\mathrm{CO}_{2}$ incubator until grown to $80 \%$ confluency prior transfection. Transient transfection was performed by the Lipofectin method as the protocol from Invitrogen. The cells were harvested $48 \mathrm{~h}$ after transfection.

Transfected cells were washed 4 times with an ice-cold phosphate-buffered saline (PBS) then lysed on ice for $30 \mathrm{~min}$ in a PBS lysis buffer [5 mM EDTA, 0.5\% NP-40, $0.1 \mathrm{mM}$ PMSF plus $10 \mu \mathrm{M}$ pepstatin $\mathrm{A}, 10 \mu \mathrm{M}$ leupeptin and $25 \mu \mathrm{g} / \mathrm{ml}$ aprotinin, purchased from Roche Co., Shanghai, P. R. China] with gentle rocking. The cells were then centrifuged at $12,000 \times g$ for $10 \mathrm{~min}$ at $4^{\circ} \mathrm{C}$ to remove the insoluble material. The supernant was collected after preclearing the lysate by protein $\mathrm{G} /$ protein $\mathrm{A}$ agarose beads for $2 \mathrm{~h}$ at $4^{\circ} \mathrm{C}$ with rotation. At the same time, the mouse anti-HA monoclonal antibody (HA mAb) or mouse anti-Myc monoclonal antibody (Myc mAb) was incubated with protein G/protein A agarose beads for $2 \mathrm{~h}$ at $4^{\circ} \mathrm{C}$, then washed 3 times with PBS and resuspended in PBS. The precleared lysate and antibody-agarose beads were mixed and incubated for $2.5 \mathrm{~h}$ at $4^{\circ} \mathrm{C}$ with gentle rocking. The immune complex was collected by centrifugation and washed 4 times with the cell lysis buffer, then solubilized in a $1 \times$ SDS buffer. The immune complex and lysate (control) were separated by $12 \%$ SDS-PAGE and electroblotted to a nitrocellulose membrane. Western blotting was performed using Myc mAb or HA $\mathrm{mAb}$ and a corresponding secondary antibody. The immunoreactive proteins were visualized using the enhanced chemiluminescence detection system that was purchased from Santa Cruz Biotechnology Inc. (Santa Cruz, USA).

Fluorescence and confocal microscopy TCP10L and MAD4 proteins were localized by immunofluorescence and confocal microscopy. The TCP1OL gene was constructed to pcDNA3.1/mycHis(-)B vector with primers (P9: 5'-GTAGAATTCATGCTGGCA GGTCAAC-3', P10: 5'-GTCAAGCTTAGGACACCCCCCC-3') to form a fusion protein with a Myc tag (TCP10L-Myc). Mad4 was constructed to the pEGFP vector with primers (P11: 5'-ACTAG ATCTATGGAGCTGAACTCCCTGC-3', P12: 5'-ATAGGATCCT TCGAGAGGGCGGGGC-3') to form the EGFP-MAD4 fusion protein.

The HEK293 cells were grown on coverslips and co-transfected, as described in "Materials and Methods". After a 48 h-transfection, the cells were fixed in $4 \%$ paraformaldehyde for $10 \mathrm{~min}$. The coverslips were washed once with TBS (50 mM Tris-HCl pH7.4, $150 \mathrm{mM} \mathrm{NaCl}$ ), then the cells were permeabilized with $0.2 \%$ Triton $\mathrm{X}-100$ for $5 \mathrm{~min}$. The slips were washed 3 times for $5 \mathrm{~min}$ each with TBS, and the cells quenched in fresh $0.1 \%$ sodium 
borohydride in TBS for $5 \mathrm{~min}$. All of the slips were blocked with a blocking buffer ( $1 \%$ horse serum, $\left.1 \% \mathrm{BSA}, 0.02 \% \mathrm{NaN}_{3}, 1 \times \mathrm{PBS}\right)$ for $1 \mathrm{~h}$, then washed for $5 \mathrm{~min}$ with TBS. Myc mAb was diluted with $1: 200(\mathrm{v} / \mathrm{v})$ in TBS (containing 1\% BSA) and centrifuged for $20 \mathrm{~min}$ at $12,000 \times g$. Stain the cells with diluted Myc mAb and incubated overnight at $4^{\circ} \mathrm{C}$. The slips were washed $3 \times 5 \mathrm{~min}$ with TBS and incubated with Cy5 that was diluted $1: 10000(\mathrm{v} / \mathrm{v})$ in TBS (containing 1\% BSA) for $45 \mathrm{~min}$ in the dark. The slips were washed $3 \times 5$ min with TBS in low lighting and the coverslips were mounted on slides for the microscopy.

\section{Results}

Identification of MAD4 as TCP10L-associate protein To identify the proteins that can interact with TCP10L, the yeast two-hybrid screening technique was used. Because the TCP1OL gene expressed specifically in the liver and testis tissues (for females, specifically in the liver), we screened the human liver cDNA Library.

We plated a pool of cells that contained $5 \times 10^{7}$ primary library transformants at a multiplicity of 4 onto the selection induction medium. Approximately 20 positive clones were obtained, among them, 5 clones could activate both the LEU2 and $L a c Z$ reporter gene only when they contained pLexA/ TCP10L. The cDNA of interest was isolated and sequenced. A sequence homology search identified the encoded proteins. All of these peptides were in-frame to the MAX dimerization protein 4 (MAD4) and contained a partial N-terminal deletion portion of MAD4. Mad4 is a known transcriptional factor gene (GenBank accession number: NM_006454). It is transcribed into a cDNA of 3773 bp and contains an openreading frame of $630 \mathrm{bp}$ that codes a peptide of 209 AA with a molecular mass of $21 \mathrm{KDa}$. The homologous proteins of MAD4 in mice belong to MAD protein family. Each member contains bHLHZip and interacts with the MAX protein through the leucine zipper domain (Ayer et al., 1993; Zervos et al., 1993; Hurlin et al., 1995; Nair and Burley 2003). The TCP10L protein contains a leucine zipper domain in its head region ( 75 to $96 \mathrm{AA}$ ), which gives rise to the hypothesis that the interaction between MAD4 and TCP10L is mediated through the leucine zipper domain. The following experiment gave us preliminary proof. The pB42AD/Mad4 was cotransformed separately with pLexA/TCP10L-H and pLexA/ TCP10L-T. The transformants were selected on an induction medium. Only the clones that were co-transformed with pB42AD/Mad4 and pLexA/TCP10L-H turned blue, indicating the interaction of these two proteins (Fig. 1). Because only the TCP10L head has the leucine zipper domain, leucine zipper domains mediate the interaction between MAD4 and TCP10L.

TCP10L co-precipitated with MAD4 To eliminate the false positive result from the yeast two-hybrid assay, the interaction between MAD4 and TCP10L was confirmed by an in vivo binding assay. The pCMV-HA/TCP10L and pCMV-

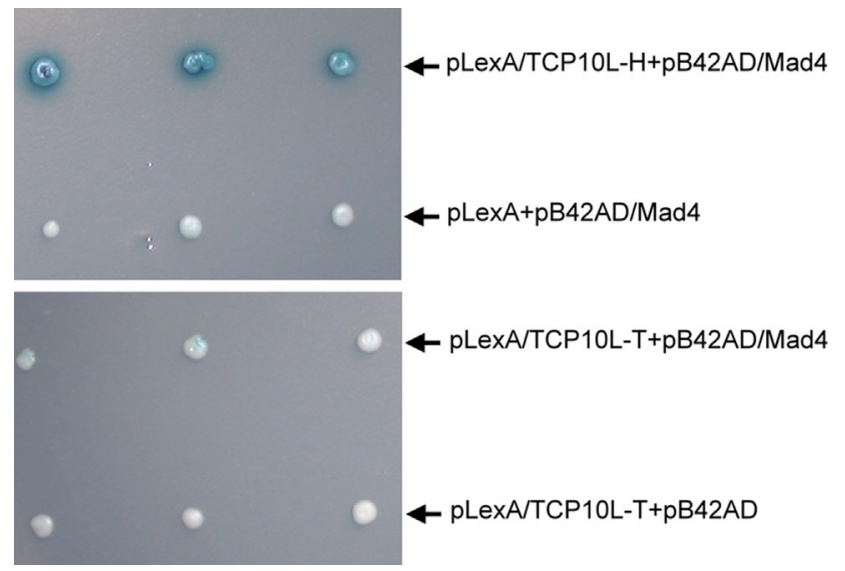

Fig. 1. Protein-protein interaction between TCP10L (head and tail) and MAD4 demonstrated in a yeast two-hybrid analysis. The yeast strain EGY48(p8op-lacZ) was co-transformed with pLexA/TCP10L-H and pB42AD/Mad4 or pLexA/TCP10L-T and $\mathrm{pB} 42 \mathrm{AD} / \mathrm{Mad} 4$ to test their interaction. Then pLexA and $\mathrm{pB} 42 \mathrm{AD} / \mathrm{Mad} 4$, pLexA/TCP10L-T, and pB42AD were separately co-transformed to serve as the controls. All of the transformants were selected on the induction medium.

Myc/Mad4 were co-transfected into HEK293 cells. Whole cell lysates were immunoprecipitated with HA mAb or Myc $\mathrm{mAb}$, followed by an immunoblot analysis using Myc mAb or HA mAb. MAD4-Myc was clearly detected in the anti-HA immunoprecipitations from the cells that were co-transfected with pCMV-HA/TCP10L and pCMV-Myc/Mad4, and for the two negative controls, pCMV-HA/TCP10L with pCMV-Myc, pCMV-HA with pCMV-Myc/Mad4, MAD4-Myc was undetectable. In addition, the MAD4-Myc that was detected in the immunoprecipitations was the same size as that in the lysate (Fig. 2A). TCP10L-HA was detected in anti-Myc immunoprecipitations from cells that were co-transfected with pCMV-HA/TCP10L and pCMV-Myc/Mad4, and undetectable in the two negative controls (Fig. 2B). Therefore, the result further provided evidence that there is a direct interaction between MAD4 and TCP10L in vivo.

Subcellular co-localization of MAD4 and TCP10L MAD4 and TCP10L can directly interact in vivo (Fig. 2), but their subcellular distribution patterns are unknown. Confocal microscope and immunofluorescence methods were used to localize their subcellular residence.

The HEK293 cells that were co-transfected with pcDNAMyc/TCP10L and pEGFP/Mad4 were harvested and processed as described in "Materials and Methods". Recombinant protein TCP10L-Myc was labeled with Cy5 waves red excitation light, while protein MAD4-EGFP produced green excitation light in the confocal microscope. MAD4 and TCP10L distributed exclusively in the nuclei of the co-transfected cells in a diffusion pattern, and were undetectable in other organelles (Fig. 3). In the merged photo, yellow spots (overlaps between green and red) were clearly 


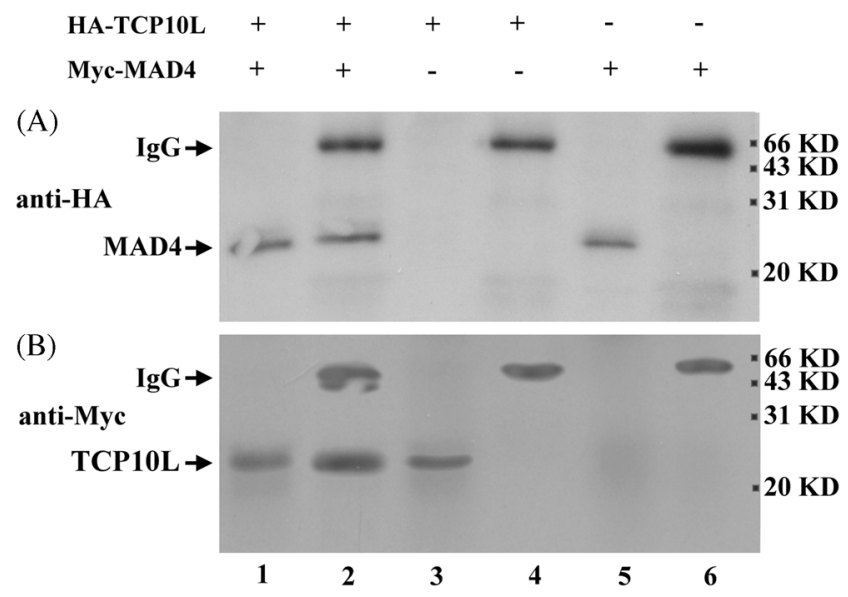

Fig. 2. Association of TCP10L with MAD4 in vivo. Interaction between TCP10L and MAD4 was assayed in the transfected HEK293 cells. (A) A lysate of the co-transfected cells was immunoprecipitated by $\mathrm{HA} \mathrm{mAb}$, followed by immunoblotting with Myc mAb. (B) A lysate of the co-transfected cells was immunoprecipitated by Myc $\mathrm{mAb}$, followed by immunoblotting with HA mAb. In lanes 1,3 , and 5, a lysate of the cotransfected cells was directly immunoblotted with Myc mAb (in A) or $\mathrm{HA} \mathrm{mAb}$ (in B). In lanes 2, 4, and 6, the lysate of the cotransfected cells was immunoprecipitated by $\mathrm{HA} \mathrm{mAb}$, then immunoblotted with $\mathrm{Myc}$ mAb (in A) or immunoprecipitated by Myc mAb, then immunoblotted with HA mAb (in B).

seen, which strongly indicates that MAD4 and TCP10L are co-localized in the nuclei of transfected cells.

\section{Discussion}

Using the yeast two-hybrid method and with TCP10L as a bait, the partner protein MAD4 was obtained. Also, we provided further evidence that the interaction between TCP10L and MAD4 is through leucine zipper motifs.
Subsequent research by immunoprecipitation and subcellular localization also confirmed this interaction. A Northern blot revealed that TCP1OL is expressed in the human liver and testis (Chen et al., 2003, in press). The TCP10L expression patterns are different from those of its homologue $T C P 10 b^{t}$ in mice, which is exclusively expressed in the testis. Since TCP10L can interact and co-localize with MAD4, the expression TCP1OL patterns may, in some way, indicate the $M A D 4$ expression in the liver and testis. But at present, there are no reports on the expression patterns of Mad4 in the human adult liver and testis. In cultured 3T3-L1cells, Mad4 is expressed in quiescent cells, which decreased upon serumstimulation and is reinduced as cells approach confluency (Pulverer et al., 2000). In mouse erythroleukemia cells, Mad4 mRNA is highly induced and persists in terminally differentiated cells (Kime and Wright, 2003), which is similar to the deduced expression pattern of Mad4 in human liver cells, since most cells in a normal liver are differentiated. During murine embryogenesis, Mad4 mRNA is primarily expressed in growth-arrested differentiating cells in the developing central nervous system and epidermis (Hurlin et al., 1995), but continuously expressed during the proliferative and differentiative phases in the developing prostrate gland (Luo et al., 2001); both show the Mad4 function on cell differentiation. Based on the previous expression analysis, we suggest that the biological functions of the interaction between TCP10L and MAD4 are to maintain the differentiation state of liver cells.

During our research on the TCP1OL expression pattern in human hepatoma tissues and their adjacent normal tissues, we found that TCP10L mRNA was down-regulated in $42 \%$ of the human hepatocellular carcinoma (HCC) cases by Northern blotting. Further examinations in 48 human HCC cases through RT-PCR confirmed this observation (personal communication). But, what is the reason for the downregulation of TCP1OL in human hepatoma tissues? Protooncogene $c-M y c$ is involved in hepatocarcinogenesis (Yaswen
(A)

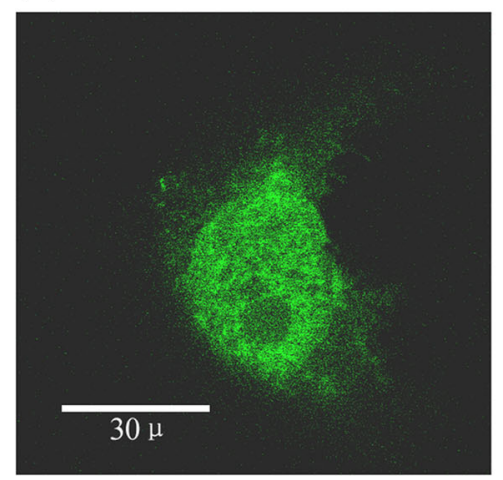

(B)

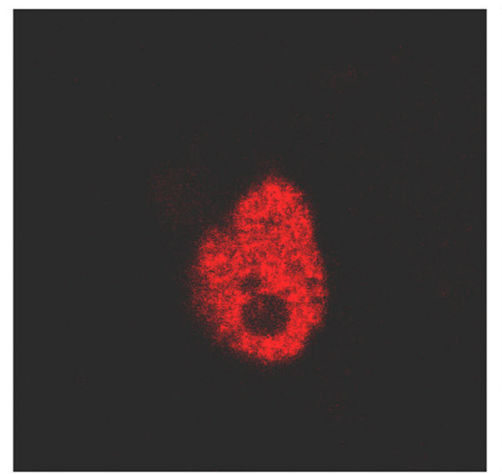

(C)

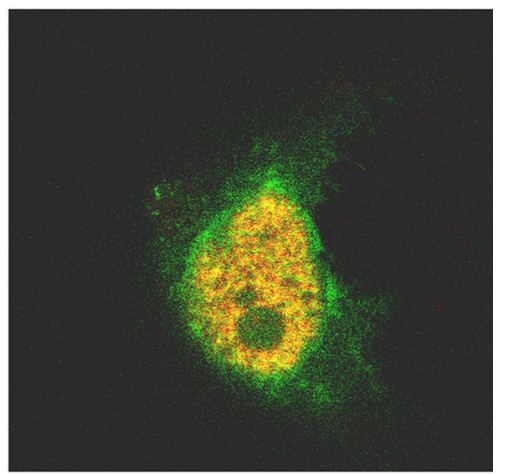

Fig. 3. Subcellular localization of TCP10L and MAD4 by confocal microscopic analysis. The HEK293 cells were co-transfected with pcDNA3.1-Myc/TCP10L and pEGFP/Mad4.The transfected cells were treated as described in "Materials and Methods". (A) The MAD4-EGFP image of the co-transfected cells. (B) The TCP10L-Myc image of the co-transfected cells. (C) The merge of A and B. In $\mathrm{A}, \mathrm{B}$, and $\mathrm{C}$ only the nucleus was visualized. 
et al., 1985; Giri and Das, 1999), and the enhanced expression has been detected in most human HCC (Zhang et al., 1990; Gan et al., 1993). Systematic detection of $c-M y c$ amplification in 400 patients with $\mathrm{HCC}$ revealed that the amplification ratio varied in patients. The amplification was low in the single nodular HCC (12\%) when compared to the multinodular HCC $(38 \%)$. In metastatic HCC, the amplification was $29 \%$ in the primary tumor and $45 \%$ in the metastatic tumor. Amplification was highest in recurrent $\mathrm{HCC}$, with a ratio of $38 \%$ in the primary tumor and $60 \%$ in the recurrent tumor (Wang et al., 2002). The up-regulation of $c-M y c$ was accompanied with the down-regulation of Mad4, because $c$ $M y c$ is one member of the transcription complex that represses the Mad4 expression (Kime and Wright, 2003). Since TCP10L interacts with MAD4, its expression may, in some way, be affected by c-Myc. Therefore, the up-regulation of $c-M y c$ may be connected to the down-regulation of TCP1OL in human HCC.

We analyzed the biological functions of the TCP10L: MAD4 complex in liver, but how are their functions exerted? TCP10L interacts with MAD4, which will block the heterodimerization between MAD4 and MAX as their interaction is also mediated by leucine zippers. But it is unknown whether the TCP10L:MAD4 complex can bind to the E-box in the same way that the MAX:MAD4 complex does. If it does, then this means that TCP10L can take the role of MAX and participate in the regulation of E-box genes in the liver and testis. If not, then TCP10L may block the suppressive effect of MAD4 on MYC functions. The presence of the TCP10L:MAD4 complex in liver cells supports the idea that the complex, with the help of other members, can bind to the E-box and regulate the corresponding genes in the liver. The Max-like protein-X (MLX) is a bHLHZip protein in mice that can take the role of MAX and selectively interact with MAD1 and MAD4 (Billin et al., 1999). TCP10L may act in a similar way. However, further study is needed to elucidate the detailed functional mechanism of the TCP10L:MAD4 complex in the liver and testis.

Acknowledgments We are grateful to Drs. Yongjun Dang, Songmin Jiang, and Chaoqun Wu for their technical assistance and helpful discussions. We also thank Drs. Youheng Wei and Yongping Cai for the gene sequencing.

\section{References}

Amati, B., Brooks, M. W., Levy, N., Littlewood, T. D., Evan, G. I. and Land, H. (1993) Oncogenic activity of the c-Myc protein requires dimerization with Max. Cell 72, 233-245.

Ayer, D. E., Kretzner, L. and Eisenman, R. N. (1993) Mad: A heterotrimeric partner for Max that antagonizes Myc transcriptional activity. Cell 72, 211-222.

Baudino, T. A. and Cleveland, J. L. (2001) The Max Network Gone Mad. Mol. Cell Biol. 21, 691-702.

Billin, A. N., Eilers, A. L., Queva, C. and Ayer, D. E. (1999) Mlx, a novel max-like bHLHZip protein that interacts with the network of transcription factors. J. Biol. Chem. 274, 3634436350.

Cebra-Thomas, J. A., Decker, C. L., Linda, C. S., Stephen, H. P. and Silver, L. M. (1991) Allele- and haploid-specific product generated by alternative splicing from a mouse $\mathrm{t}$ complex responder locus candidate. Nature 349, 239-241.

Dang, C. V. (1999) c-Myc target genes involved in cell growth, apoptosis, and metabolism. Mol. Cell Biol. 19, 1-11.

Ewulonu, U. K., Buratynski, T. J. and Schimenti, J. C. (1993) Functional and molecular characterization of the transcriptional regulatory region of $T c p-10 b^{t}$, a testis-expressed gene from the $\mathrm{t}$ complex responder locus. Development 117, 89-95.

Facchini, L. M. and Penn, L. Z. (1998) The molecular role of Myc in growth and transformation: recent discoveries lead to new insights. FASEB J. 12, 633-651.

Gan, F. Y., Gesell, M. S., Alousi, M. and Luk, G. D. (1993) Analysis of ODC and c-myc gene expression in hepatocellular carcinoma by in situ hybridization and immunohistochemistry. J. Histochem. Cytochem. 41, 1185-1196.

Giri, R. K. and Das, B. R. (1999) Mutational activation of c-Ha-ras oncogene or cancer promotion by Phenobarbital upregulates the transcription of c-jun and c-myc oncogene in N-nitrosodiethylamine-mediated hepatocarcinogenesis. Hepatology Research 16, $1-11$.

Henriksson, M. and Luscher, B. (1996) Proteins of the Myc network: essential regulators of cell growth and differentiation. Adv. Cancer Res. 68, 109-182.

Hurlin, P. J., Queva, C., Koskinen, P. J., Steingrimsson, E., Ayer, D. E., Copeland, N. G., Jenkins, N. A. and Eisenman, R. N. (1995) Mad3 and Mad4: novel Max-interacting transcriptional repressors that suppress c-myc dependent transformation and are expressed during neural and epidermal differentiation. EMBO J. 14, 5646-5659.

Kime, L. and Wright, S. C. (2003) Mad4 is regulated by a transcriptional repressor complex that contains Miz-1 and cMyc. Biochem. J. 370, 291-298.

Luo, Q., Harmon, E., Timms, B. G. and Dretzner, L. (2001) Novel expression patterns of the $\mathrm{myc} / \mathrm{max} / \mathrm{mad}$ transcription factor network in developing murine prostate gland. J. Urol. 166, 1071-1077.

Luscher B. and Larsson L. G. (1999) The basic region /helix-loophelix/leucine zipper domain of Myc proto-oncoproteins: function and regulation. Oncogene 18, 2955-2966.

Nair, S. K. and Burley, S. K. (2003) X-ray structures of myc-max and mad-max recognizing DNA: molecular bases of regulation by proto-oncogenic transcription factors. Cell 112, 193-205.

Pisani, P., Parkin, D. M. and Ferlay, J. (1993) Estimate of the worldwide mortality from eighteen major cancers in 1985. Implications for prevention and projections of future burden. Int. J. Cancer 55, 891-903.

Pisani, P., Parkin, D. M., Munoz, N. and Ferlay, J. (1997) Cancer and infection: estimate of the attributable fraction in 1990. Cancer Epidemiol. Biomarkers Prev. 6, 387-400.

Pulverer, B., Sommer, A., McArthur, G. A., Eisenman, R. N. and Luscher, B. (2000) Analysis of Myc/Max/Mad network members in adipogeneis: inhibition of the proliferative burst and differentiation by ectopically expressed Mad1. J. Cell. Physiol. 183, 399-410.

Schimenti, J., Cebra-Thomas, J. A., Decker, C. L., Islam, S. D., 
Pilder, S. H. and Silver, L. M. (1988) A candidate gene family for the mouse $t$ complex responder (Tcr) locus responsible for haploid effects on sperm function. Cell 55, 71-78.

Smith, M. J., Charron-Prochownik, D. C. and Prochownik, E. V. (1990) The leucine zipper of c-Myc is required for full inhibition of erythroleukemia differentiation. Mol. Cell. Biol. 10, 5333-5339.

Wang, Y., Wu, M. C., Sham, J., Zhang, W. G., Wu, W. Q. and Guan, X. Y. (2002) Prognostic significance of c-myc and AIB1 amplification in hepatocellular carcinoma. Cancer 95, 23462352
Yaswen, P., Goyette, M., Shank, P. R. and Fausto, N. (1985) Expression of c-Ki-ras, c-Ha-ras and c-myc in specific cell types during hepatocarcinogenesis. Mol. Cell Biol. 5, 780-786.

Zhang, X. K., Huang, D. P., Qiu, D. K. and Chiu, J. F. (1990) The expression of c-myc and c-N-ras in human cirrhotic livers, hepatocellular carcinomas and liver tissue surrounding the tumors. Oncogene 5, 909-914.

Zervos, A. S., Gyuris, J. and Brent, R. (1993) Mxi1, a protein that specifically interacts with Max to bind Myc-Max recognition sites. Cell 72, 223-232. 